\title{
ARTICLE
}

\author{
Genetics and Genomics
}

\section{Primary constitutional MLH1 epimutations: a focal epigenetic}

\section{event}

\author{
Estela Dámaso ${ }^{1}$, Adela Castillejo ${ }^{2}$, María del Mar Arias ${ }^{3}$, Julia Canet-Hermida ${ }^{1}$, Matilde Navarro ${ }^{1}$, Jesús del Valle ${ }^{1}$, Olga Campos $^{1}$, \\ Anna Fernández ${ }^{1}$, Fátima Marín ${ }^{1}$, Daniela Turchetti ${ }^{4}$, Juan de Dios García-Díaz ${ }^{5}$, Conxi Lázaro ${ }^{1}$, Maurizio Genuardi ${ }^{6}$, Daniel Rueda ${ }^{7}$, \\ Ángel Alonso ${ }^{3}$, Jose Luis Soto ${ }^{2,8}$, Megan Hitchins ${ }^{9}$, Marta Pineda ${ }^{1}$ and Gabriel Capellá ${ }^{1}$
}

\begin{abstract}
BACKGROUND: Constitutional MLH1 epimutations are characterised by monoallelic methylation of the MLH1 promoter throughout normal tissues, accompanied by allele-specific silencing. The mechanism underlying primary $M L H 1$ epimutations is currently unknown. The aim of this study was to perform an in-depth characterisation of constitutional MLH1 epimutations targeting the aberrantly methylated region around $M L H 1$ and other genomic loci.

METHODS: Twelve MLH1 epimutation carriers, 61 Lynch syndrome patients, and 41 healthy controls, were analysed by Infinium $450 \mathrm{~K}$ array. Targeted molecular techniques were used to characterise the $M L H 1$ epimutation carriers and their inheritance pattern. RESULTS: No nucleotide or structural variants were identified in-cis on the epimutated allele in 10 carriers, in which intergenerational methylation erasure was demonstrated in two, suggesting primary type of epimutation. CNVs outside the MLH1 locus were found in two cases. EPM2AIP1-MLH1 CpG island was identified as the sole differentially methylated region in MLH1 epimutation carriers compared to controls.

CONCLUSION: Primary constitutional MLH1 epimutations arise as a focal epigenetic event at the EPM2AIP1-MLH1 CpG island in the absence of cis-acting genetic variants. Further molecular characterisation is needed to elucidate the mechanistic basis of $M L H 1$ epimutations and their heritability/reversibility.
\end{abstract}

British Journal of Cancer (2018) 119:978-987; https://doi.org/10.1038/s41416-018-0019-8

\section{INTRODUCTION}

Lynch syndrome (LS) is characterised by an increased risk for colorectal cancer (CRC) and cancers of the endometrium, ovary, stomach, small intestine, hepatobiliary tract, urinary tract, brain, and skin $^{1}$. LS is caused by a germline genetic variant within a mismatch repair (MMR) gene, MLH1 (MutL homolog 1), MSH2 (MutS Homolog 2), MSH6 (MutS homolog 6) or PSM2 (PMS1 Homolog 2), or a terminal deletion of EPCAM (Epithelial Cell Adhesion Molecule gene) with consequent epigenetic inactivation of $M S H 2$. In a small proportion of LS patients the cancer predisposition is caused by a constitutional epimutation of $M L H 1$, in which one allele of the CpG island promoter is aberrantly hypermethylated throughout normal tissues with associated loss-of-expression from this allele ${ }^{2}$.

Two types of constitutional MLH1 epimutation have been defined: secondary, which are linked in-cis to a genetic alteration and follow an autosomal dominant pattern of inheritance ${ }^{3-10}$; and primary, which occur in the absence of any apparent linked sequence change, typically arise de novo $0^{6,11-16}$, and demonstrate null $^{6,11,13,17}$ or non-Mendelian inheritance ${ }^{9,11,18}$. To date, 75 index cases with a constitutional $\mathrm{MLH1}$ epimutation have been reported $^{19,20}$, accounting for $2-3 \%$ of mutation negative cases with suspected LS whose tumors are MLH1-deficient ${ }^{21}$. Most of these $(66 / 75)$ have been considered primary ${ }^{19,20}$. The available evidence from these cases suggests that constitutional MLH1 epimutations cause a severe LS phenotype, including a young age of cancer onset and multiple primary tumors.

Previous studies on constitutional $M L H 1$ epimutation have focused on the role of this molecular defect in cancer causation by confirming the presence of $M L H 1$ promoter methylation and corresponding transcriptional silencing within normal tissues, refining the selection criteria for patients warranting screening for it, and the inheritance patterns within families. It has been proposed that primary $\mathrm{MLH} 1$ epimutations arise in the germline or early stages of embryonic development, since they are monoallelic and soma-

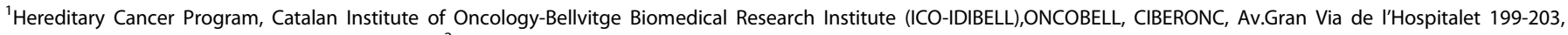

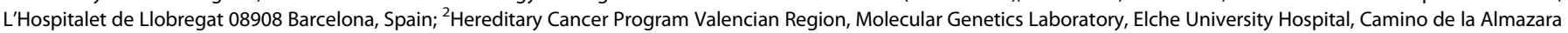

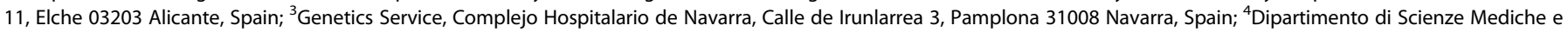

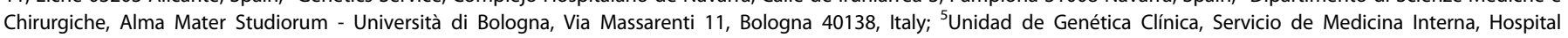

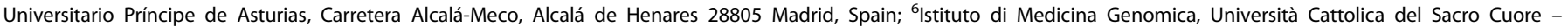

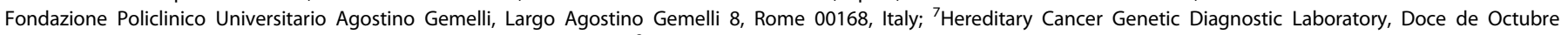

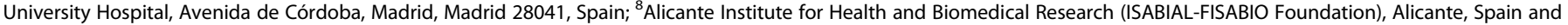
${ }^{9}$ Department of Medicine, Division of Oncology, Stanford University, 1291 Welch Road, Stanford 94305 California, USA

Correspondence: Marta Pineda (mpineda@iconcologia.net) or Gabriel Capellá (gcapella@iconcologia.net)

These authors contributed equally: Marta Pineda, Gabriel Capellá.

Joint senior authors: Marta Pineda, Gabriel Capellá.

Received: 28 July 2017 Revised: 20 November 2017 Accepted: 27 November 2017

Published online: 4 October 2018 
wide, but frequently exhibit mosaic methylation and expression loss ${ }^{2}$. However, no comprehensive studies have been undertaken to elucidate the mechanism(s) underlying primary $M L H 1$ epimutations, such as whether they are localized within the $M L H 1$ locus due to a focal defect, or if additional genes are concomitantly affected due to widespread epigenetic perturbation. The main aim of our study was to perform an in-depth characterisation of the methylation profile in cases with a confirmed constitutional MLH1 epimutation to define the extent of the aberrantly methylated region around $M L H 1$, as well as other loci on a genome-wide scale. The results from this study contribute to the understanding of primary constitutional $\mathrm{MLH} 1$ epimutations by showing that the methylation error occurs in a localized manner.

\section{PATIENTS AND METHODS}

Patients and samples

Twelve Caucasian MLH1 epimutation carriers were recruited from the west Mediterranean area, among which eight were previously reported $13,22,23$. The four new cases were referred from Complejo Hospitalario de Navarra and Doce de Octubre University Hospital due to the identification of $M L H 1$ methylation in blood by MS-MLPA. The original numerical code for each proband was maintained from previous publications; however, a prefix corresponding to the referral center (below) was added (Table S1). Clinico-pathological data collected included age at cancer diagnosis, tumor location, immunohistochemistry of MMR proteins in tumors, and $\mathrm{MLH} 1$ methylation status in tumor and blood DNA (Table S1). Forty-one healthy controls (selected to appropriately match the patients by age, race, and geographic location), 61 LS patients with confirmed genetic mutations (21 MLH1, 28 MSH2, 9 MSH6, 3 PMS2), and four CRC patients whose tumors demonstrated somatic $\mathrm{MLH1}$-methylation, were included as controls (Table S2). Written informed consent was obtained from all individuals, and the ethics committee of the respective hospitals approved the study.

Samples of peripheral blood leukocytes (PBL) DNA were collected from biobanks at the Catalan Institute of Oncology (ICO), Elche University Hospital (HGUE), Valencian Biobank Network (IBSP-CV), Complejo Hospitalario de Navarra (CHN), Doce de Octubre University Hospital (H12O), and Policlinico S.OrsolaMalpighi (M). Lymphoblastoid cell lines were established from the B lymphocytes of $M L H 1$ epimutation carriers by standard EBVtransformation using supernatant from the B95.5 cell lines, as described in Supplementary Methods. Colorectal tumors and distal normal mucosa were collected as formalin-fixed paraffin embedded (FFPE) tissues when available (Table S2).

For each FFPE specimen, $10-20 \times 10-\mu \mathrm{m}$ sections were cut from a single-representative block per case, using macrodissection with a scalpel if needed to enrich for tumor cells. After deparaffinization using Deparaffinization Solution (Qiagen, Hilden, Germany), DNA was isolated using the QIAmp DNA FFPE Tissue Kit (Qiagen) according to the manufacturer's instructions.

Methylation analyses

The initial detection or confirmation of the presence of $M L H 1$ methylation in each proband and consenting family members was performed by methylation-specific multiplex ligation-dependent probe amplification (MS-MLPA) on genomic DNA from PBL. For all other methylation analyses, DNA was subject to sodium bisulphite modification using the EZ DNA Methylation Kit (Zymo Research), according to the manufacturer's instructions.

MS-MLPA. The presence of methylation at the MLH1 promoter was detected and confirmed using $100 \mathrm{ng}$ of PBL DNA using the SALSA MLPA ME011 Mismatch Repair genes probemix (MRCHolland), according to the manufacturer's instructions. DNA from the RKO CRC cell line was used as the $M L H 1$ methylation-positive control. The amplification products were run on an ABI Prism 3130
DNA sequencer and analysed using GeneMapper v4.0 (Applied Biosystems).

Clonal bisulfite sequencing. Clonal bisulfite sequencing of fragments of the $M L H 1$ promoter was used to determine the allelic methylation profile in bisulfite modified PBL DNA from $\mathrm{MLH} 1$ epimutation carriers who were found to be heterozygous for a promoter variant. Primers were used to amplify specific promoter regions encompassing each promoter variant (Table S3), and PCR products were cloned in E. coli cells using the TOPO TA cloning kit (Invitrogen) to separate the amplicons into individual alleles. The inserts from at least 24 individual colonies were sequenced using plasmid vector primers. Additional methodological details are provided in Supplementary Methods. The methylation status at individual CpG site and the allele of each heterozygous variant within each promoter fragment was determined using SeqMan software (DNASTAR) or Mutation Surveyor (SoftGenetics).

Methylation array analysis. PBL DNA samples (1000 ng) from patients and controls, and 500-1000 ng of available FFPE colorectal cancer DNA and distal normal colon tissue DNA, were randomized within 96-well plates. For internal quality control, in vitro methylated and unmethylated DNAs were included in each batch.

For quality control purposes, PBL DNA integrity was evaluated by agarose gel electrophoresis and spectrophotometry. DNAs from FFPE samples were analysed by qPCR using the Infinium FFPE QC kit (Illumina), and samples showing a delta-Ct value below five were restored using the Infinium HD FFPE Restore kit (Illumina), following the manufacturer's instructions.

Quantity of $1000 \mathrm{ng}$ PBL and $500 \mathrm{ng}$ FFPE DNAs were bisulfite converted using the EZ DNA Methylation Kit (Zymo Research), according to the manufacturer's instructions. To determine the efficiency of the bisulfite conversion, a predetermined genomic region was evaluated by Sanger sequencing in the methylated and unmethylated controls and one sample from each batch.

Genome-wide methylation profiling was performed using the Infinium Human Methylation $450 \mathrm{~K}$ Beadchip (Illumina), which interrogates the methylation status of $485,764 \mathrm{CpG}$ sites across the genome. After hybridization, sample scanning was performed using the HiScan platform (Illumina), which has a laser scanner with two colors $(532 \mathrm{~nm} / 660 \mathrm{~nm})$. The relative intensity of each dye was analysed using GenomeStudio software (Methylation Module). For each analysed CpG site, a $\beta$-value was obtained depending on the florescence intensity. $\beta$ measures took values between 0 (unmethylated) and 1 (fully methylated). Analysis for batch effects was performed using RnBeads software (Max-PlanckInstitut Informatik). Group comparisons and statistical analyses (based on differentially methylated CpG sites, CpG islands, promoters, genes and tiling) were also performed using RnBeads software. CpG methylation was visualized by Integrative Genome Viewer (Broad Institute). GRCh37/hg19 was used as the reference genome.

The data discussed in this publication are accessible through GEO Series accession number GSE107353 (https://www.ncbi.nlm. nih.gov/geo/query/acc.cgi?acc = GSE107353).

Analysis of Alu elements. The sequence encompassing the MLH1EPM2AIP1 CpG island identified as the differentially methylated region (DMR) in carriers of an $\mathrm{MLH} 1$ epimutation was screened for repetitive elements by the RepeatMasker Web Server (Institute for Systems Biology) using "Cross_match" as the search engine. No repetitive elements were found in this DMR. Downstream of the DMR, Alu elements were found in $M L H 1$ intron 1, as previously described $^{24}$. The methylation status of these Alu elements was tested in PBL DNA from three $\mathrm{MLH} 1$ epimutation carriers, one healthy control and the RKO cell line by bisulfite sequencing of the PCR fragments designated p24 (8 CpGs from Alu 2) and p25 (1 
CpG from Alu 3), as previously described ${ }^{24}$. Amplification of the region p10 (control region, including $6 \mathrm{CpGs}$, unmethylated in normal tissues and methylated in cancer tissues) was performed using methylation-specific primers. Primers and conditions are summarized in Table S3.

Mutational analyses

Screen of the EPM2AIP1-MLH1 CPG island for point mutations. The EPM2AIP1-MLH1 CpG island is defined by its CpG content as the region Chr3:37,034,229-37,035,355 by the UCSC genome browser (CpG:93). Screening for promoter variants was performed by amplification of two overlapping PCR fragments followed by direct sequencing. Primers and PCR conditions are provided in Table S3. Sequencing was performed using the BigDye Terminator v.3.1 Sequencing Kit (Applied Biosystems). Mutation Surveyor (SoftGenetics) was used for sequence visualization.

Detection of structural variants around the MLH1 locus. A screen for the presence of structural alterations was performed on $\mathrm{PBL}$ DNA from $M L H 1$ epimutation carriers using a custom highdefinition $\mathrm{CGH}$ array designed with eArray Software (Agilent Technologies) and manufactured by Agilent's SurePrint oligo technology. This comprised 15,000 probes encompassing the MLH1 locus (region Chr3:36,450,000-37,900,000 within cytoband 3 22.2), with an average probe spacing of $100 \mathrm{bp}$ intervals. Bioinformatics analysis was performed in $\mathrm{R}$ using the 2.15 .12 Bioconductor statistical packages. Results were visualized on CytoGenomics Software (Agilent Technologies).

In silico functional evaluation of the genetic variants identified PROMO 3.0 software ${ }^{25,26}$ was used to predict any changes in transcription factor binding between wild-type alleles and promoter variants. Only human transcription factors were considered and $5 \%$ was selected as maximum matrix dissimilarity rate.

Structural variants were compared with those previously reported in the Database of Genomic Variants ${ }^{27}$, using chromosomal coordinates.

MLH1 allelic expression analyses

For allelic expression analyses at the $M L H 1$ single-nucleotide polymorphism (SNP) rs1799977 (c.655A>G), the relative levels of the two alleles were determined in genomic DNA and CDNA by single-nucleotide primer extension (SNuPE) as previously described $^{13}$ (see Supplementary Methods for details).

\section{Haplotype analysis}

Haplotype analysis was performed using four intragenic MLH1 SNPs rs1800734, rs9876116, rs1799977, and rs4234259, and seven microsatellite markers, D3S1609, D3S1612, D3S2369, D3S1611, D3S3623, D3S1298, and D3S3564, spanning $12 \mathrm{Mb}$ around MLH1, as previously described ${ }^{28}$. To deduce the methylation-associated haplotype, intrafamilial segregation analysis was performed under the assumption that the number of crossovers between adjacent markers was minimal.

\section{RESULTS}

Validation of newly identified $M L H 1$ epimutations and associated functional impact

MLH1 promoter methylation was confirmed by MS-MLPA in blood from newly identified epimutation carriers (Table 1). Three of them presented methylation values higher than $40 \%$, whereas case CHN_2 displayed evidence of epigenetic mosaicism.

The frequent SNP rs1799977 (c.655G>A) within MLH1 exon 8 was used to determine the effect of the epimutation on the allelic transcriptional activity of $\mathrm{MLH1}$ in five carriers who were heterozygous for this SNP. Monoallelic expression of the G allele was identified in lymphocytes from case CHN_1, indicating complete loss-of-expression from the epimutant $A$ allele. In cases CHN_2 and H12O-A, partial transcriptional silencing was observed in lymphoblastoid cell lines derived from patient lymphocytes, consistent with the observed methylation mosaicism (Table 1). CHN_2 had reduced expression of the A allele with allele-specific expression (ASE) 0.53, and H120-A had reduced expression of the $\mathrm{G}$ allele, with ASE 0.79 (Table 1). Loss-of-expression of the epimutant A allele was previously demonstrated in cases ICO_1 and $M 60^{13,23}$. The remaining cases were homozygotes for the polymorphism, thus allelic expression analyses could not be performed in them.

Genetic characterisation of the $M L H 1$ locus

Since secondary constitutional $M L H 1$ epimutations are linked to rare variants or rearrangements involving the EPM2AIP1-MLH1 promoter, mutational analysis of the whole-CpG island encompassing the EPM2AIP1-MLH1 promoter was performed. The common SNP rs1800734 (c.-93G >A) was identified in five of the 12 patients (42\%) and rs34566456 (c. $-593 \mathrm{G}>\mathrm{C}$ ) in case ICO_34. Additionally, case HGUE 5 was heterozygous for a novel variant $c$. -234_-236delCTT (Figure S1). In silico, this is located within the binding region for the TFII-I and GATA-1 transcription factors, but these were predicted to be unaffected by the variant. No other variants, including $c .[-27 \mathrm{C}>\mathrm{A} ; \mathrm{c} .85 \mathrm{G}>\mathrm{T}]^{3}$ - were identified within the $M L H 1$ promoter region.

Screening for structural variants surrounding the $M L H 1$ locus (Chr3:36,450,000-37,900,000) identified three small deletions in two of the 11 patients analysed, for whom sufficient DNA was available (Figure S2, Table 1). Two deletions outside the differentially methylated $\mathrm{CpG}$ island were identified in case HGUE_2: one $15 \mathrm{~Kb}$ in size located upstream of the $M L H 1$ gene (Chr3:36,798,479-36,813,411) and the other of $19 \mathrm{~Kb}$ in size located downstream (Chr3:37,486,324-37,505,162) (Figure S2A). Neither of these two deletions has previously been described as copy number variations in the DGV database (Table S4). In case HGUE_1, a $20 \mathrm{~Kb}$ deletion was identified upstream of the MLH1 gene (Chr3:36,396,587-36,416,879) (Figure S4B). This region appears to be prone to copy number variations in the control reference group (DGV database), as shown in Table S4. In both cases (HGUE_1 and HGUE_2), validation of these deletions using a second method was not possible due to sample depletion.

Determination of the methylation-associated allele (MAA) Clonal bisulfite sequencing was performed on PBL DNA of probands harboring a heterozygous variant within the $M L H 1$ promoter region to determine the MAA. Dense monoallelic MLH1 methylation was linked to the $G$ allele at SNP rs1800734 (c. $-93 \mathrm{G}>\mathrm{A}$ ) in cases H12O-A and HGUE 2, and to the allele $A$ in patients CHN_1 and HGUE_5 (Table 1). In case HGUE_5, who was heterozygous for both the novel heterozygous variant c.-234 $-236 \mathrm{del}$ and the c. $-93 \mathrm{G}>\mathrm{A}$ SNP, methylation was linked to the non-deleted and c.-93A alleles, and the [c.-234_-236del; c.-93G] haplotype was unmethylated (Figure S3). This finding indicated the c.-234_-236del variant was not causally linked to the epimutation in HGUE_5. In patient ICO_34, methylation was linked to the $G$ allele at SNP rs34566456 (c. $-593 G>C$ ) with $~ 38 \%$ of $\mathrm{G}$ alleles methylated, indicating mosaicism (Table 1), consistent our prior findings using other methods ${ }^{13}$.

Inheritance pattern of the epimutant alleles and designation of $M L H 1$ epimutation as likely primary or secondary

To investigate the inheritance pattern of the genetic allele harboring the epimutation, the $M L H 1$ promoter methylation status and haplotypes within a $12 \mathrm{Mb}$ around $\mathrm{MLH} 1$ were determined in available PBL DNA from the first-degree relatives of epimutation carriers (Table S5). MS-MLPA analysis showed no evidence of MLH1 methylation in any of the relatives tested from any family, 


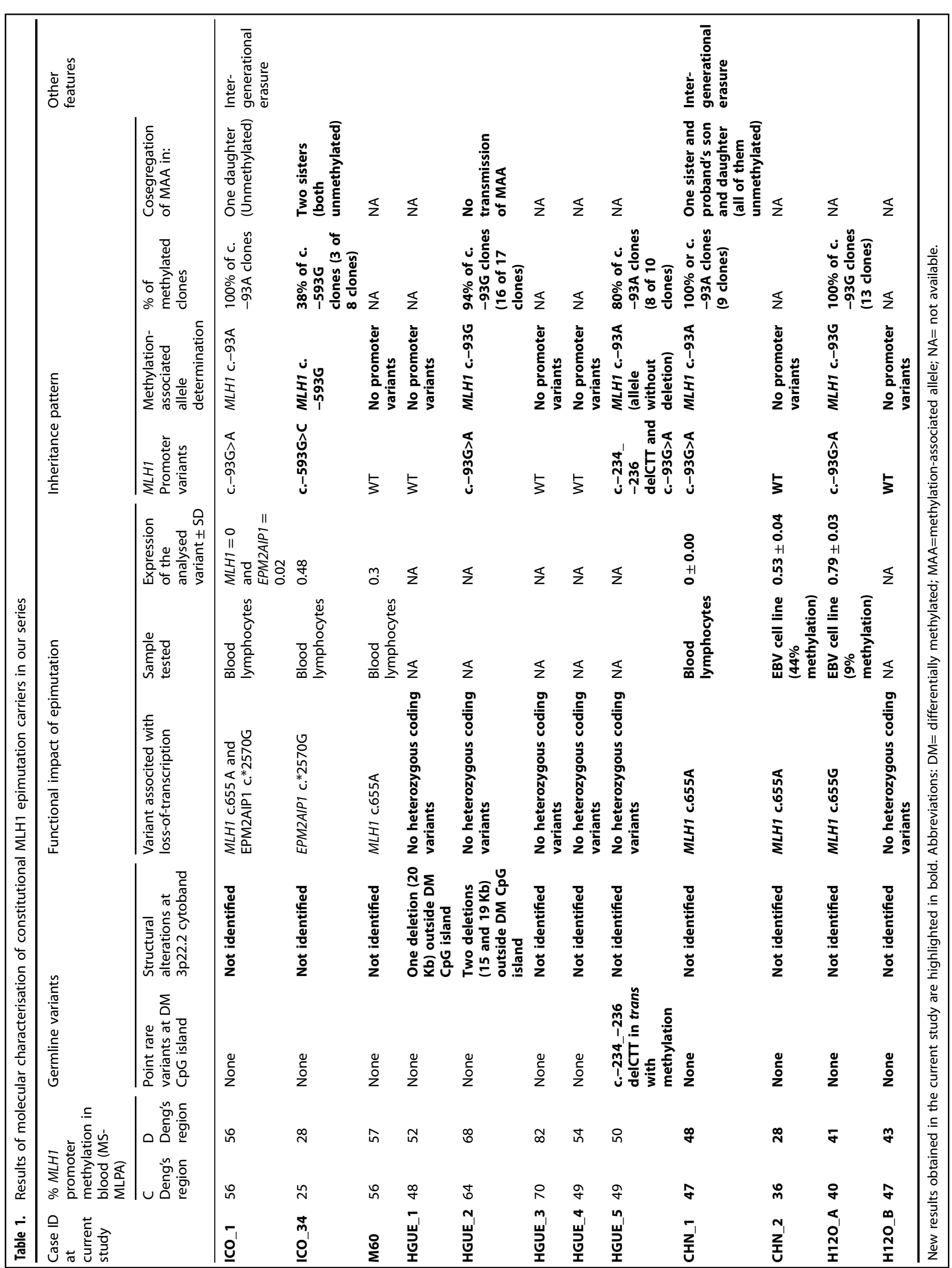



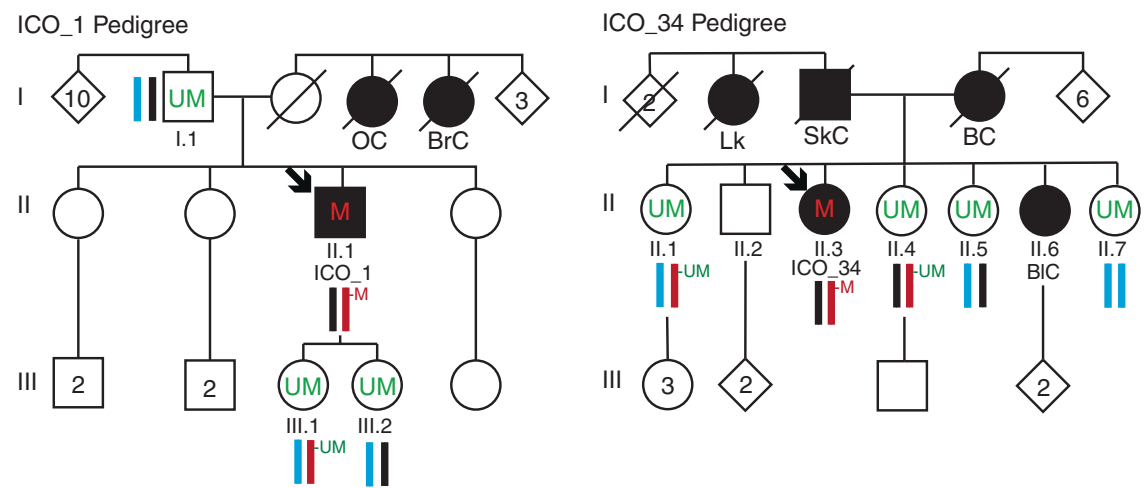

HGUE_2 Pedigree

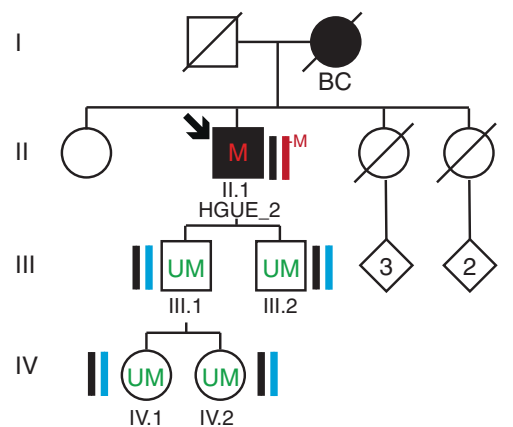

CHN_1 Pedigree

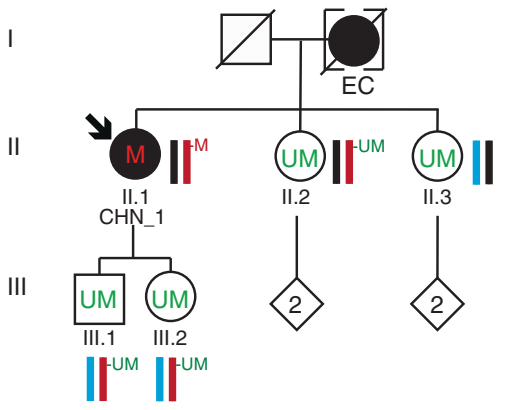

Fig. 1 Pedigrees of the $M L H 1$ epimutation cases showing that the presence of the epimutation does not co-segregate with any genetic allele. Circles, females; squares, males; filled, cancer affected. Cancer localization (CRC, colorectal cancer; OC, ovarian cancer; EC, endometrial cancer; $\mathrm{BC}$, breast cancer; Lk, leukaemia; BrC, brain cancer; PC, prostate cancer) and age at diagnosis are indicated. Epimutation carriers are indicated by an arrow. Generations are indicated on the left margin in Roman numerals and analysed relatives are identified by numbers. Haplotypes are schematised by sticks: Red = methylation-associated allele (MAA), Black = haplotype also carried by the proband but not associated with the epimutation, Blue $=$ un-informative haplotypes. The presence of methylation $(M)$ or its absence (UM) is indicated on the red MAA haplotypes for those individuals tested)

thus no case of transmission of an epimutation was observed among the family members tested (Figs. 1, 2). Haplotype analyses revealed that the genetic allele bearing the epimutation (in the proband) was shared in unmethylated form by two sisters of case ICO_34, suggesting a de novo occurrence of the epimutation in proband ICO_34; one sister and two offspring of case CHN_1, suggesting de novo occurrence of the epimutation in proband CHN_1 and indicating inter-generational erasure of the epimutation in her offspring (Fig. 1). The inter-generational erasure newly observed in case CHN_1 herein, and previously observed in case ICO_ $1^{13}$, allowed us to definitively classify the epimutation in these two cases as primary. In six additional cases, the absence of methylation in the relatives tested, and/or the lack of a family history of cancer, also suggested their epimutation was of the primary type.

In patient HGUE_2, although clonal bisulphite sequencing showed monoallelic methylation of the c.-93A allele, we were unable to determine whether this was linked in-cis to either or both of the $15 \mathrm{~Kb}$ and $19 \mathrm{~Kb}$ deletions flanking $M L H 1$ that were also identified in this patient. The MAA was not transmitted to either of his offspring, hence this family was non-informative for whether the type of epimutation carried by this proband was primary or secondary. Similarly, in case HGUE_1, we were unable to determine whether the epimutation was linked in-cis to the 20 $\mathrm{Kb}$ deletion identified upstream of $M L H 1$, hence whether the deletion may have been causally related.

Global epigenetic characterisation of $M L H 1$ constitutional epimutations

In order to evaluate whether aberrant methylation was restricted to $M L H 1$ or extended to other loci across the genome in $M L H 1$ epimutation carriers, methylation array analysis was performed using the Infinium $450 \mathrm{k}$ array in PBL DNA. This revealed that the MLH1-EPM2AIP1 promoter CpG island was the sole differentially methylated region (DMR) in $M L H 1$ epimutation carriers when compared to healthy controls (false discovery rate (FDR) adjusted $p$-value $=2.45 \mathrm{E}-42$ ) (Fig. 3a). The same result was obtained when MLH1 epimutation carriers were compared to LS cases (FDR adjusted $p$-value $=3.45 \mathrm{E}-61$ ) (Fig. $3 \mathrm{~b}$ ), and the subset of LS cases with germline $\mathrm{MLH} 1$ mutation (FDR adjusted $p$-value $=6.41 \mathrm{E}-21$ ) (Fig. 3c). Accordingly, the same analysis identified $M L H 1$ and EPM2AIP1 as the unique differentially methylated promoters in MLH1 epimutation carriers compared to healthy controls (EPM2AIP1 FDR adjusted $p$-value $=2.21 \mathrm{E}-43, M L H 1$ FDR adjusted $p$-value $=2.19 \mathrm{E}-35)$, LS patients (EPM2AIP1 FDR adjusted $p$-value $=5.07 \mathrm{E}-61, M L H 1$ FDR adjusted $p$-value $=9.23 \mathrm{E}-51$ ), and $M L H 1$ mutated LS cases (EPM2AIP1 FDR adjusted $p$-value $=8.41 \mathrm{E}-21$, MLH1 FDR adjusted $p$-value $=1.51 \mathrm{E}-16$ ) (Fig. 3).

A deeper analysis at the $M L H 1$ promoter region showed that the methylation levels in PBL from constitutional epimutation carriers spread across the entire CpG island, MLH1 exon 1 and the first part of intron 1 (Fig. 4). The CpG island shores were also affected by hypermethylation, represented by probes at positions chr3: $37,033,791$ and chr3: $37,035,400$ at the boundaries of the $1.6 \mathrm{~Kb}$ DMR. Of note, no other common DMR were found in the genomewide methylation analysis among the carriers of a MLH1 constitutional epimutation.

The DMR identified in the PBL of $M L H 1$ epimutation carriers was also present in subject-matched normal colonic mucosa and colorectal cancer (CRC) (when available for analysis). Although methylation levels across the DMR were marginally higher in CRC tissue than normal tissues, this did not reach statistical 


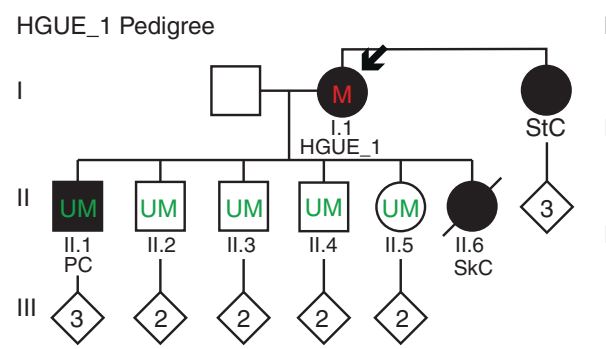

HGUE_3 Pedigree
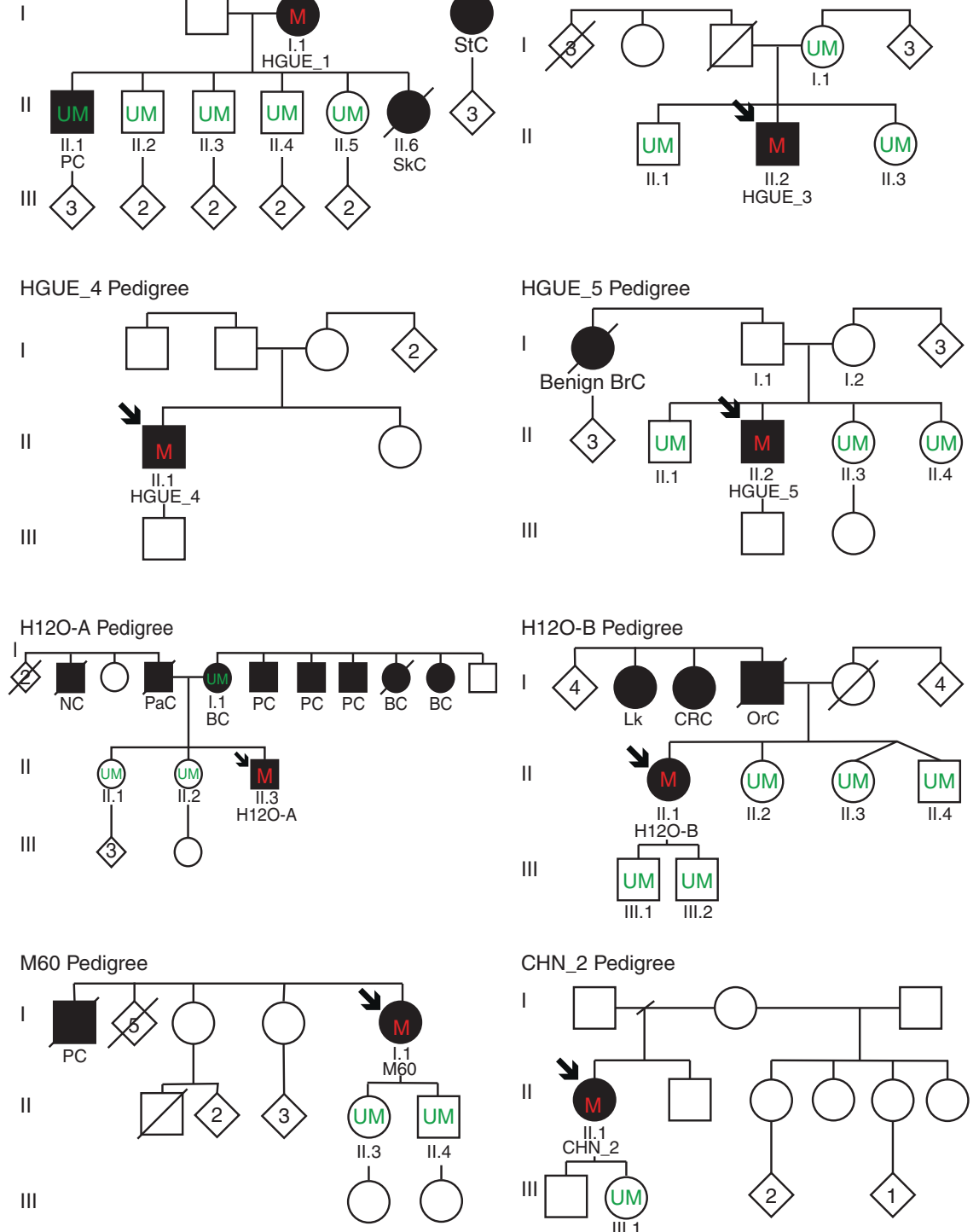

Fig. 2 Pedigrees of the $M L H 1$ epimutation carriers not included in Fig. 1.Circles, females; squares, males; filled, cancer affected. Cancer localization (CRC, colorectal cancer; BC, breast cancer; Lk, leukaemia; BrC, brain cancer; PC, prostate cancer; SkC, skin cancer; GC, gastric cancer; OrC, oral cancer; $\mathrm{BC}$, breast cancer; $\mathrm{PaC}$, pancreatic cancer; $\mathrm{NC}$, neck cancer) and age at diagnosis are indicated. Epimutation carriers are indicated by an arrow. Generations are indicated on the left margin in Roman numerals and analysed relatives are identified by numbers. The presence of methylation (M) or its absence (UM) is indicated if tested

significance, suggesting that methylation is not a common second hit in these tumors (Fig. 5a). Similarly, no significant differences were found in the levels of methylation among the tissues tested from mutation-positive LS cases (Fig. 5b).

In normal colonic mucosa tissue, a lack of $M L H 1$ methylation was similarly observed in the LS control group and the sporadic cases with somatic MLH1 methylation in their tumors (Figure S3A). By contrast, in tumor tissue, the sporadic MLH1-methylated CRC cases showed a more extensive DMR spanning $\approx 50 \mathrm{~Kb}$ (from chr3:36,985,516 to chr3:37,035,399), within which four CpG sites located at chr3:36,986,513; 36,986,532; 36,986,555, and 36,986,642 were differentially methylated only in the sporadic CRC (FDR adjusted $p$-value $<0.01$ ) (Figure S3B and S3C).

Methylation state of Alu elements

In order to evaluate whether demethylation of $M L H 1$ intron 1 Alu sequences is associated with constitutional epimutation, as has been suggested for sporadic MSI tumors ${ }^{24}$, bisulfite sequencing analysis was performed in PBL DNA from $3 \mathrm{MLH} 1$ epimutation carriers (cases ICO_1, ICO_34, and CHN_1), one healthy control and the RKO cell line. As previously described, partial demethylation was observed in Alu 3 of RKO cells (Figure S4). By contrast, the intron 1 Alu CpGs analysed were found to be fully methylated in carriers of a constitutional $M L H 1$ epimutation and a healthy control (Figure S4), arguing against demethylation of these nearby Alu sequences as a causal mechanism for constitutional epimutation.

\section{DISCUSSION}

A comprehensive genetic and epigenetic analysis has provided a deeper and broader molecular characterisation of 12 cases with constitutional $\mathrm{MLH} 1$ epimutation than previously undertaken. The series, which includes 4 novel cases, accounts for $16 \%$ of all index 
A Constitutional $M L H 1$ epimutation carriers vs. Healthy controls
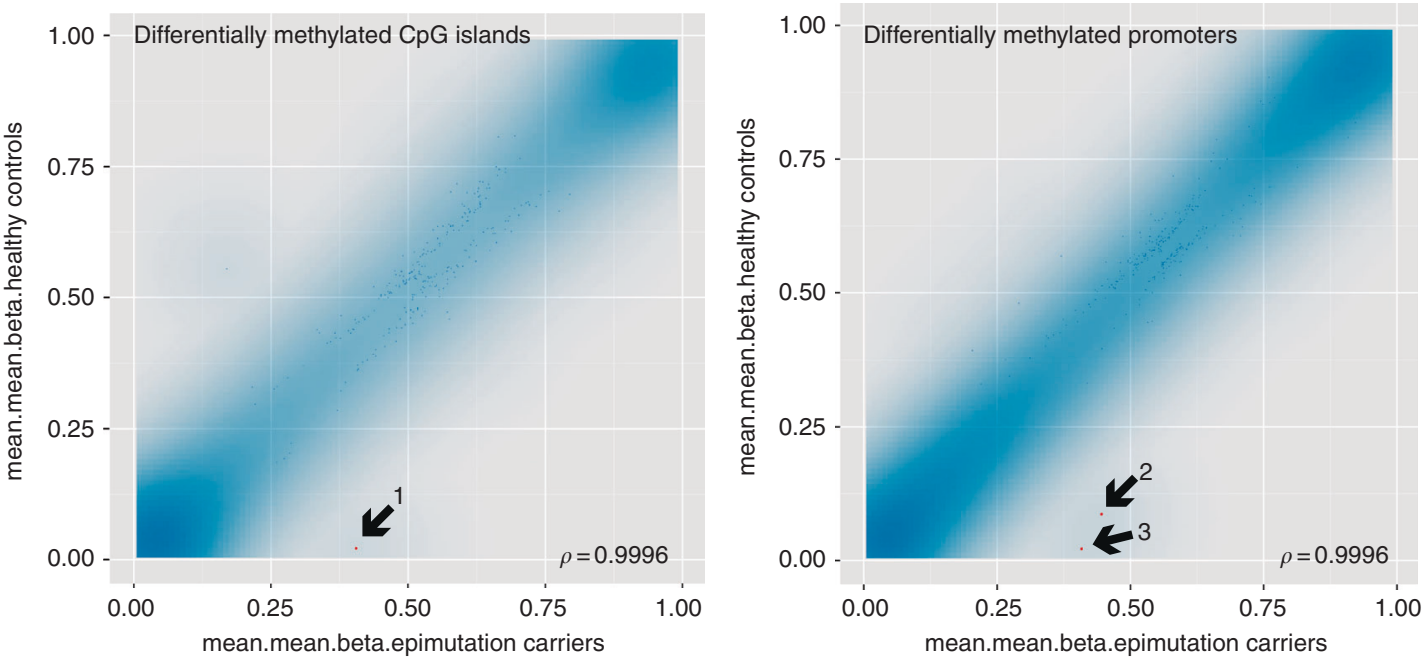

B Constitutional MLH1 epimutation carriers vs. mutation-positive Lynch syndrome patients
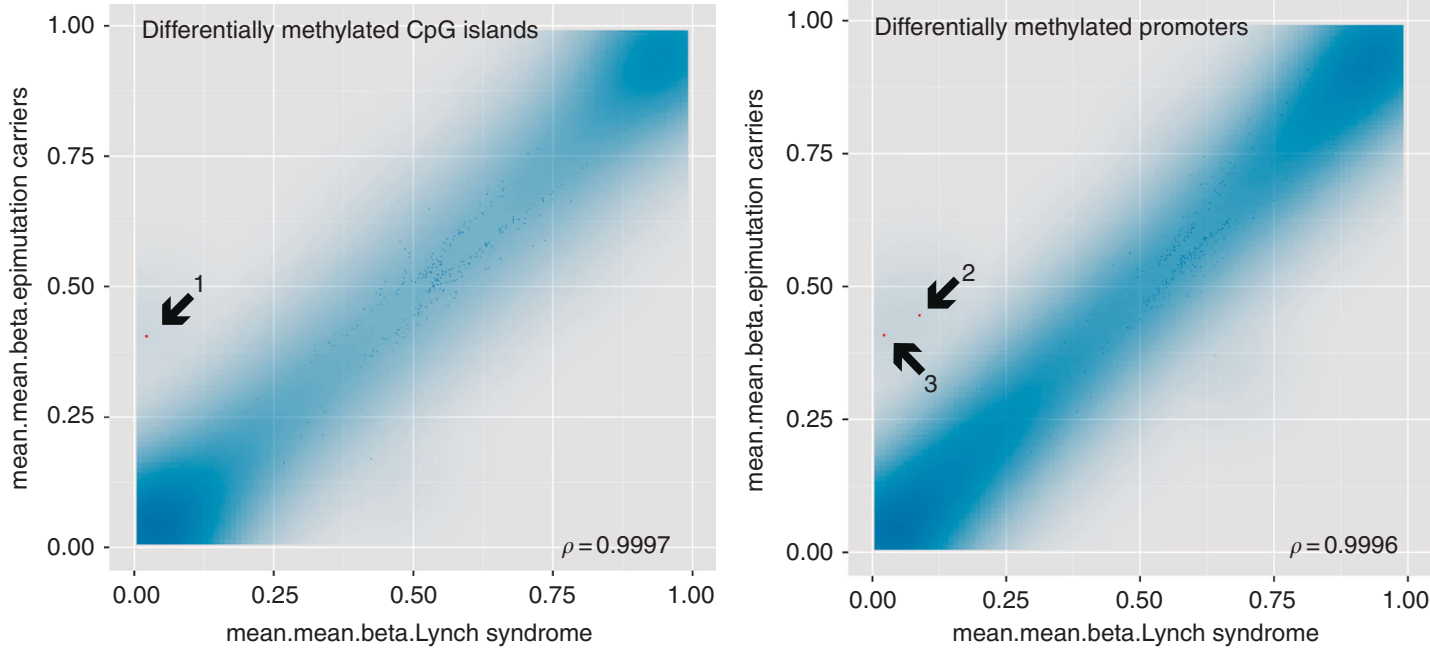

C

FDR adjusted $p$-values

\begin{tabular}{|l|c|c|c|}
\hline Group comparison & $\begin{array}{c}{ }^{1} \text { EPM2AIP1-MLH1 CpG island } \\
\text { Chr3:37,034,229-37,035,356 }\end{array}$ & ${ }^{2}$ MLH1 promoter & ${ }^{3}$ EMP2AIP1 promoter \\
\hline $\begin{array}{l}\text { Epimutation carriers } \\
\text { vs. Healthy controls }\end{array}$ & $2.45 \mathrm{E}-42$ & $2.19 \mathrm{E}-35$ & $2.21 \mathrm{E}-43$ \\
\hline $\begin{array}{l}\text { Epimutation carriers } \\
\text { vs. mut-positive LS patients }\end{array}$ & $3.45 \mathrm{E}-61$ & $9.23 \mathrm{E}-51$ & $5.07 \mathrm{E}-61$ \\
\hline $\begin{array}{l}\text { Epimutation carriers } \\
\text { vs } M L H 1 \text {-mut LS patients }\end{array}$ & $6.41 \mathrm{E}-21$ & $1.51 \mathrm{E}-16$ & $8.41 \mathrm{E}-21$ \\
\hline
\end{tabular}

Fig. 3 Scatterplot of the normalized mean $\beta$-values obtained using the Infinium $450 \mathrm{k}$ Human Methylation array to identify differentially methylated CpG islands and promoters in blood DNA from constitutional MLH1 epimutation carriers against healthy controls a and Lynch syndrome patients with a germline sequence mutation $\mathbf{b}$. The transparency corresponds to point density. One \% of the points in the sparsest populated plot regions are drawn explicitly. The colored points represent differentially methylated CpG islands (depicted as arrow \#1) and promoters (arrows \#2 and \#3). FDR adjusted $p$-values of differentially methylated regions are summarized at the bottom $\mathbf{c}$.

cases of constitutional $\mathrm{MLH1}$ epimutation reported to date (Table S6). Our findings suggest that the epimutations in at least 10 of these cases are primary based on the following: (i) the absence of rare in-cis genetic aberrations within the MLH1 CpG island, (ii) the lack of familial cancer history and the presence of the MAA in an unmethylated state in siblings and/or parents that point to a de novo occurrence of the epimutation in the probands
(Table S1 and S5); and (iii) the demonstration in two cases of the inter-generational erasure of the epimutation in offspring who inherited the MAA. Interestingly, few studies of $M L H 1$ epimutation that have involved family members from more than one generation have ever been undertaken, but among these, both inter-generational inheritance and erasure of the epimutation between the proband and offspring have been described 

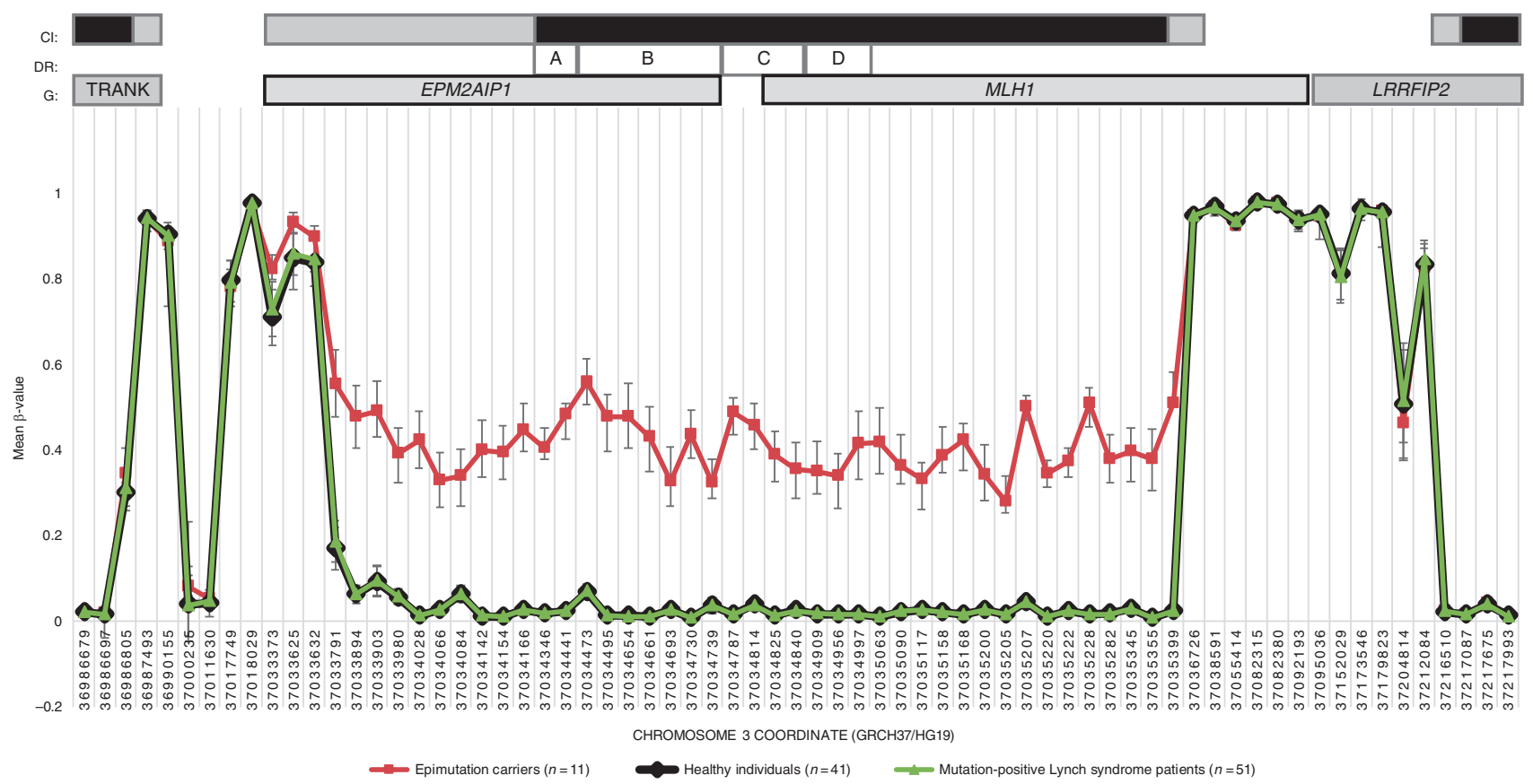

Fig. 4 Representation of the differentially methylated region across the $M L H 1$ locus in blood DNA from constitutional MLH1 epimutation carriers (red, $n=11$ ), Lynch syndrome mutation carriers (green, $n=61$ ) and healthy controls (black, $n=41$ ). $\beta$-values obtained from Infinium $450 \mathrm{k}$ Human Methylation array analysis are displayed against the genomic coordinate for each CpG site interrogated. The relative locations of the CpG sites are not drawn to scale. CpG sites located between Chr3:37,033,791-37,035,399 coordinates had FDR adjusted $p$-values between $2.5 \mathrm{E}-7$ and $1.2 \mathrm{E}-59$ for comparisons of $\mathrm{MLH1}$ epimutation carriers against Lynch syndrome patients or healthy controls. Above, CpG islands (CI) are represented as black rectangles and their shores are represented in grey. Location of Deng's promoter regions (DR) is indicated as white rectangles A-D. Genes $(G)$ containing the displayed CpG sites are represented as grey rectangles. Ensembl GRCh37 was taken as reference for gene coordinates

(Table S5). The two cases of inter-generational erasure, we describe herein provide further evidence of the reversibility of this defect between generations (Table S5, Fig. 1).

In two cases, HGUE_1 and HGUE_2, deletions external to $M L H 1$ were identified by $\mathrm{aCGH}$. However, we were unable to confirm the presence of these deletions by other methods due to the lack of sample availability. Also, we could not determine whether the deletions occurred on the MAA. Noteworthy, secondary MLH1 epimutations have been reported with concomitant genetic alterations in-cis, including promoter deletions, a promoter single-nucleotide variant, and a large duplication encompassing MLH1 and neighboring genes ${ }^{3-10,29}$. These cases have displayed autosomal dominant inheritance of the $M L H 1$ epimutation with faithful segregation of the methylation with the genetic alteration. Therefore, although we cannot completely rule out that deletions are causative of the epimutation in cases HGUE_1 and HGUE_2, the absence of family history and methylation in blood from firstdegree relatives, led us to propose to classify them as "suspected primary" (Table S6).

Transcriptional silencing usually correlates with methylation levels (Table S6). In case H12O-A, differences in the values of methylation and transcriptional silencing could be found depending upon the sample tested. The lymphoblastoid cell line displayed less methylation than that observed in blood, in line with a previous observation ${ }^{30}$. These results highlight the limitations of using lymphoblastoid cell lines for epigeneticbased analyses, although this may be a necessity when primary tissue samples from patients are limited.

The array-based genome-wide methylation analysis performed in PBL DNA demonstrated that a region of $1.6 \mathrm{~Kb}$ (Chr3:37,033,791-37,035,400)—encompassing the shared MLH1EPM2AIP1 CpG island and its corresponding shores- was the sole DMR unique to the group of $M L H 1$ epimutation carriers, as compared to mutation-positive LS patients or healthy controls. For cases HGUE_1 and HGUE_2, classified as suspected primary epimutations the same 1.6 Kb DMR was identified. No differences in the length of the DMR were observed in the normal colon mucosa or CRC tissue among the MLH1 epimutation carriers.

The absence of any other DMR in the MLH1 epimutation carrier group suggests that the constitutional epimutation is caused by a shared focal event, since it spans the very same region in all patients tested. Of note, our preliminary analysis does not suggest a genetic cause. Also, we have provided evidence against $\mathrm{MLH} 1$ intron 1 Alu sequence demethylation as a causal mechanism for constitutional MLH1 epimutation.

Interestingly, the extent of the DMR observed (consistently in all three tissue types analysed) in $M L H 1$ epimutation carriers was narrower than that the observed in sporadic MLH1-methylated CRC. Although our cases of sporadic MLH1-methylated CRC were few $(n=4)$, the extent of their methylation $(50 \mathrm{~Kb})$ is consistent with tumors with available MSI and methylation data from TCGA project $(n=43)$ (Figure S5).

While the causal mechanism of primary constitutional $\mathrm{MLH} 1$ epimutations remains unknown, it may be speculated that it acts in the very early stages of embryonic development ${ }^{2}$. MLH1 de novo methylation has been induced in vitro in human embryonic stem cells suggesting that pluripotency is a mandatory requirement for establishing the epimutation ${ }^{31}$. The detection of non-methylated clones on the MAA in distinct patients, including those with methylation levels close to $50 \%$ (Table S5), suggests that if an epimutation is established in the germline, some alleles may lose the methylation during the demethylation phase that occurs between zygote formation and the preimplantation embryogenesis ${ }^{2}$. The methylated allele could behave as a "leaky" allele when selfpropagating the $M L H 1$ epimutation within individual cells.

Our results show that aberrant methylation of the $\mathrm{MLH} 1$ promoter is targeted, as opposed to disseminated, since we found no other common DMRs among the MLH1 epimutation 


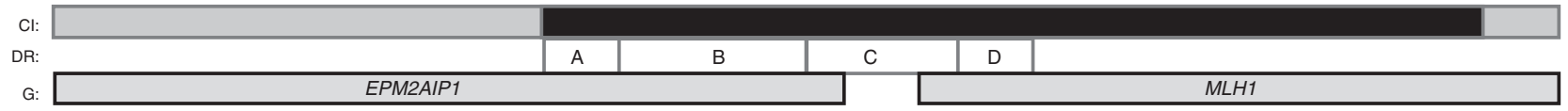

A

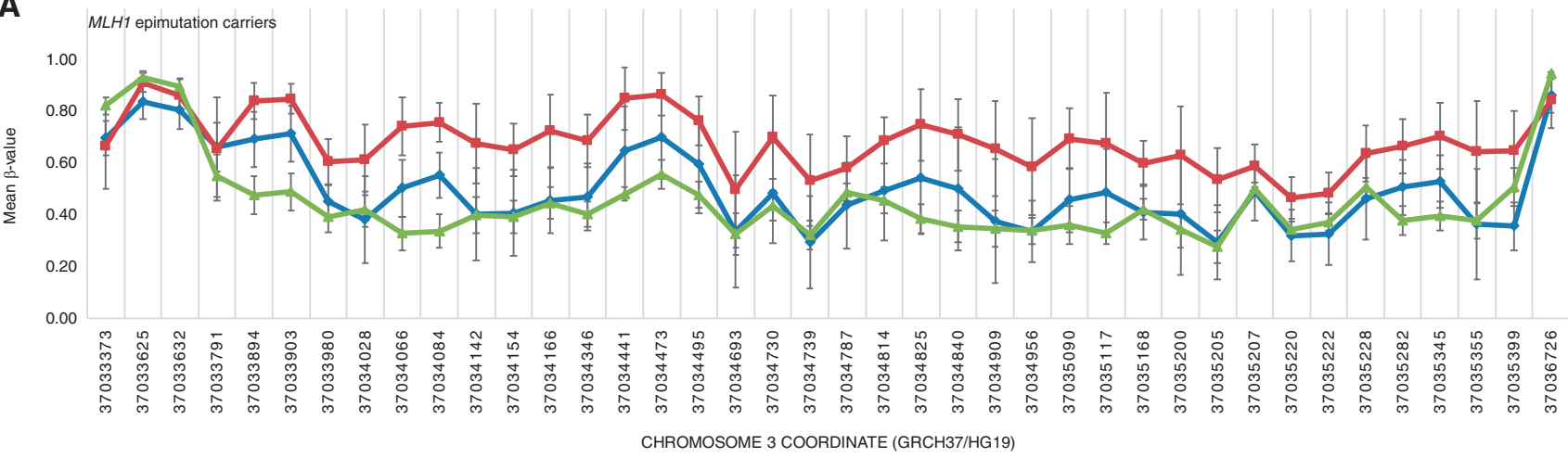

B

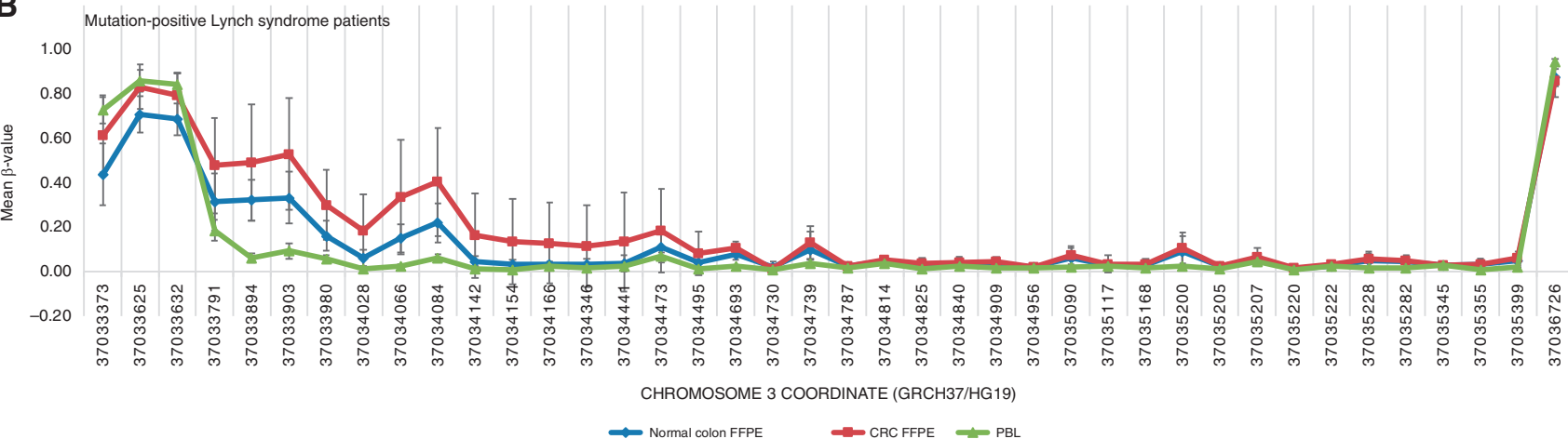

Fig. 5 Representation of differentially methylated region in blood, normal colonic mucosa, and colorectal cancer tissue in constitutional $M L H 1$ epimutation patients $\mathbf{a}$ and mutation-positive Lynch syndrome patients $\mathbf{b}$. $\beta$-values obtained after global methylation analysis are displayed against CpG sites' coordinate location. The location of the CpG sites is not drawn to scale. Above, EMP2AIP1-MLH1 CpG island (CI) is represented as black rectangle and their shores are represented in grey. Location of Deng's promoter regions (DR) are indicated as white rectangles A-D. Genes (G) including displayed CpG sites are represented as grey rectangles. Ensembl GRCh37 was taken as reference for gene coordinates.

carriers. This suggests that a primary epimutation is not derived from a trans-event or a cellular perturbation during germline or embryonic development. Further studies are needed to fully understand the mechanistic basis of $\mathrm{MLH} 1$ constitutional epimutations and their heritability/reversibility. Ongoing mechanistic studies should be focused on specific interactions of epigenetic effectors with the $M L H 1$ locus, paying special attention to those acting in the germline or during early embryonic development.

\section{ACKNOWLEDGEMENTS}

We are indebted to the patients and their families. We thank all members of the involved Genetic Counseling Cancer Units and Valencian Biobank Network. We thank Lauro Sumoy and Gabriel Rech for their support with methylation array data analysis, Miguel Àngel Peinado and Mireia Jordà for a critical revision of the manuscript. This work was funded by the Spanish Ministry of Economy and Competitiveness and cofunded by FEDER funds -a way to build Europe- (grants SAF2012-33636 and SAF2015-68016-R), Instituto de Salud Carlos III (grant PI16/00563), CIBERONC, RTICC Network (RD12/0036/0031 and RD12/0036/0008), the Spanish Association Against Cancer (080253), the Government of Catalonia (grant 2014SGR338) and Fundación Mutua Madrileña (grant AP114252013). This article is based upon work from COST Action BM1206, supported by COST (European Cooperation in Science and Technology, www.cost.eu). E.D. was supported by a grant from the Spanish Ministry of Economy and Competitiveness.

\section{ADDITIONAL INFORMATION}

Supplementary information is available for this paper at https://doi.org/10.1038/ s41416-018-0019-8.
Conflict of interest: The authors declare that they have no conflict of interest.

\section{REFERENCES}

1. Lynch, H. T., Snyder, C. L., Shaw, T. G., Heinen, C. D. \& Hitchins, M. P. Milestones of Lynch syndrome: 1895-2015. Nat. Rev. Cancer 15, 181-194 (2015).

2. Hitchins, M. P. Constitutional epimutation as a mechanism for cancer causality and heritability? Nat. Rev. Cancer 15, 625-634 (2015).

3. Hitchins Megan, P. et al. Dominantly inherited constitutional epigenetic silecancer-affected family is linked to a single nucleotide variant within tncing of MLH1 in a cancer-affected family is linked to a single nucleotide variant within the 5UTR. Cancer Cell 20, 200-213 (2011).

4. Kwok, C.-T. et al. The MLH1 c.-27C >A and c.85G > T variants are linked to dominantly inherited MLH1 epimutation and are borne on a European ancestral haplotype. Eur. J. Hum. Genet. 22, 617-624 (2014).

5. Morak, M. et al. Biallelic MLH1 SNP CDNA expression or constitutional promoter methylation can hide genomic rearrangements causing Lynch syndrome. J. Med. Genet. 48, 513-519 (2011).

6. Morak, M. et al. Further evidence for heritability of an epimutation in one of 12 cases with MLH1 promoter methylation in blood cells clinically displaying HNPCC. Eur. J. Hum. Genet. 16, 804-811 (2008).

7. Renkonen, E. et al. Altered expression of MLH1, MSH2, and MSH6 in predisposition to hereditary nonpolyposis colorectal cancer. J. Clin. Oncol. 21, 3629-3637 (2003).

8. Cini, G. et al. Concomitant mutation and epimutation of the MLH1 gene in a Lynch syndrome family. Carcinogenesis 36, 452-458 (2015).

9. Ward, R. L., Dobbins, T., Lindor, N. M., Rapkins, R. W. \& Hitchins, M. P. Identification of constitutional MLH1 epimutations and promoter variants in colorectal cancer patients from the Colon Cancer Family Registry. Genet. Med. 15, 25-35 (2013). 
10. Gylling, A. et al. Large genomic rearrangements and germline epimutations in Lynch syndrome. Int. J. Cancer 124, 2333-2340 (2009).

11. Hitchins, M. P. et al. Inheritance of a cancer-associated MLH1 germ-line epimutation. N Engl. J. Med. 356, 697-705 (2007).

12. Niessen, R. C. et al. Germline hypermethylation of MLH1 and EPCAM deletions are a frequent cause of Lynch syndrome. Genes Chromosomes Cancer 48, 737-744 (2009).

13. Pineda, M. et al. MLH1 methylation screening is effective in identifying epimutation carriers. Eur. J. Hum. Genet. 20, 1256-1264 (2012).

14. Valle, L. et al. MLH1 germline epimutations in selected patients with early-onset non-polyposis colorectal cancer. Clin. Genet. 71, 232-237 (2007).

15. Goel, A. et al. De novo constitutional MLH1 epimutations confer early-onset colorectal cancer in two new sporadic Lynch syndrome cases, with derivation of the epimutation on the paternal allele in one. Int. J. Cancer 128, 869-878 (2011).

16. Hitchins, $M$. et al. MLH1 germline epimutations as a factor in hereditary nonpolyposis colorectal cancer. Gastroenterology 129, 1392-1399 (2005).

17. Suter, C. M., Martin, D. I. K. \& Ward, R. L. Germline epimutation of MLH1 in individuals with multiple cancers. Nat. Genet. 36, 497-501 (2004).

18. Sloane, M. A., Nunez, A. C. \& Packham, D. et al. Mosaic epigenetic inheritance as a cause of early-onset colorectal cancer. JAMA Oncol. 1, 953-957 (2015).

19. Hitchins, M. P. Finding the needle in a haystack: identification of cases of Lynch syndrome with MLH1 epimutation. Fam. Cancer 15, 413-422 (2016).

20. Takeda, T. et al. Methylation analysis of DNA mismatch repair genes using DNA derived from the peripheral blood of patients with endometrial cancer: epimutation in endometrial carcinogenesis. Genes 7, 86 (2016).

21. Peltomäki, P. Update on Lynch syndrome genomics. Fam. Cancer 15, 385-393 (2016).

22. Castillejo, A. et al. Prevalence of MLH1 constitutional epimutations as a cause of Lynch syndrome in unselected versus selected consecutive series of patients with colorectal cancer. J. Med. Genet. 52, 498-502 (2015).

23. Crucianelli, F. et al. MLH1 constitutional and somatic methylation in patients with MLH1 negative tumors fulfilling the revised Bethesda criteria. Epigenetics 9, 1431-1438 (2014).

24. Wang, X. et al. Spreading of Alu methylation to the promoter of the MLH1 gene in gastrointestinal cancer. PLoS ONE 6, e25913 (2011).
25. Messeguer, X. et al. PROMO: detection of known transcription regulatory elements using species-tailored searches. Bioinformatics 18, 333-334 (2002).

26. Farré, D. et al. Identification of patterns in biological sequences at the ALGGEN server: PROMO and MALGEN. Nucleic Acids Res. 31, 3651-3653 (2003).

27. MacDonald, J. R., Ziman, R., Yuen, R. K. C., Feuk, L. \& Scherer, S. W. The Database of Genomic Variants: a curated collection of structural variation in the human genome. Nucleic Acids Res. 42(D1), D986-D992 (2014).

28. Borràs, E. et al. MLH1 founder mutations with moderate penetrance in Spanish Lynch syndrome families. Cancer Res. 70, 7379-7391 (2010).

29. Crépin, M. et al. Evidence of constitutional MLH1 epimutation associated to transgenerational inheritance of cancer susceptibility. Hum. Mutat. 33, 180-188 (2012).

30. Kwok, C.-T., Ward, R. L., Hawkins, N. J. \& Hitchins, M. P. Detection of allelic imbalance in MLH1 expression by pyrosequencing serves as a tool for the identification of germline defects in Lynch syndrome. Fam. Cancer 9, 345-356 (2010).

31. Takahashi, Y. et al. Integration of CpG-free DNA induces de novo methylation of CpG islands in pluripotent stem cells. Science 356, 503-508 (2017).

(i) Open Access This article is licensed under a Creative Commons cc) Attribution-NonCommercial-ShareAlike 4.0 International License, which permits any non-commercial use, sharing, adaptation, distribution and reproduction in any medium or format, as long as you give appropriate credit to the original author(s) and the source, provide a link to the Creative Commons license, and indicate if changes were made. If you remix, transform, or build upon this article or a part thereof, you must distribute your contributions under the same license as the original. The images or other third party material in this article are included in the article's Creative Commons license, unless indicated otherwise in a credit line to the material. If material is not included in the article's Creative Commons license and your intended use is not permitted by statutory regulation or exceeds the permitted use, you will need to obtain permission directly from the copyright holder. To view a copy of this license, visit http://creativecommons.org/licenses/by-nc-sa/4.0/.

(c) The Author(s) 2018 